\title{
Characterising bonded joints with a thick and flexible adhesive layer. Part 2: modelling and prediction of structural joint responses
}

\author{
Hasegawa ${ }^{1} \mathrm{~K}$, Crocombe $^{1}$ AD, Coppuck ${ }^{2} \mathrm{~F}$, Jewel $^{2} \mathrm{D}$ and Maher $^{2} \mathrm{~S}$ \\ ${ }^{1}$ Mechanical Engineering Sciences, University of Surrey, GU2 7XH, UK \\ ${ }^{2}$ Gordon Murray Design, Shalford, GU4 8EP, UK \\ * Corresponding author Tel: +44(0)1483689194, Email: a.crocombe@surrey.ac.uk
}

\begin{abstract}
Adhesively bonded structural joints have increasingly found applications in automotive primary structures, joining dissimilar lighter-weight materials. Low-modulus rubbery adhesives are attracting rising interest as an alternative to conventional rigid structural adhesives due to benefits such as the excellent impact resistance they provide. In this pair of papers, the mechanical behaviour of a rubbery adhesive and the bonded joints to be used in a lightweight automobile structure have been investigated, both experimentally and numerically. In this (part 2) paper, progressive damage FE modelling using cohesive elements is presented to predict the structural response of peel and lap shear specimens that were representative of the vehicle joints. The cohesive parameters that matched the load-displacement curves of fracture testing presented in part 1 were determined and used, without modification in subsequent modelling of the representative joints. The numerical predictions of these joints correlated well with the measured experimental load-displacement and damage growth data. Based on the results, it has been demonstrated that the modelling approach presented is applicable to bonded joints with a highly compliant, thick adhesive layer.
\end{abstract}

Keywords: Polyurethane (A), Fracture Toughness (C), Finite element stress analysis (C), cohesive zone model (D), strength prediction

\section{Introduction}

Bonded joints using flexible adhesives have attracted growing attention for more demanding applications such as automotive structural bonding due to their excellent impact resistance capabilities. Their high deformability allows sufficient load transfer through the joints under local high deformation incurred during an impact event as well as providing energy absorption. Improved fatigue resistance is another advantage envisaged since more uniform stress distribution compared to that for rigid adhesives can be ensured. In order to further facilitate the application of such adhesives, it is expected that their unique mechanical behaviour be fully characterised and the structural response of representative bonded joints be quantitatively predicted.

Part 1 of the paper [1] has described the experimental program which characterised the fracture behaviour and determined reliable fracture energies applicable to bonded joints using a rubbery polyurethane adhesive system with bondlines as thick as $3 \mathrm{~mm}$. It was shown that, by designing the substrate geometry to provide sufficient flexibility, the frequently used double cantilever beam (DCB) method for mode I and single leg bending (SLB) for a mixed mode (mode mixity $\left(G_{I}: G_{I I}\right)$ of $\left.4: 3\right)$ generated consistent crack growth within the adhesive layer and provided reasonable and consistent fracture energies. However, as the mode II component becomes dominant, standard testing techniques such as end notch flexure (ENF), which rely on flexural strain energy of substrates as the driving force for crack propagation, have been found to be impractical for such compliant adhesive layers. Instead, a cracked thick adherend shear test (TAST) type specimen in combination with FE analysis was used to obtain the mode II fracture energy. 
Progressive damage modelling using cohesive elements has become increasingly popular as a tool to predict the structural response of bonded joints. It has been claimed that this method has great potential in that it is capable of predicting the whole mechanical response of structural bonded joints from damage initiation through to final failure [2]. A number of studies have been reported predicting joint behaviour under quasi static loading. Yang et al. [3-5] has carried out a series of studies on predicting the mechanical behaviour of bonded joints with yielding in the substrates using FE models with cohesive elements. It has been demonstrated that the mode I and mode II cohesive parameters calibrated with a double cantilever beam joint and a torsion test of bonded butt joints, respectively, are applicable to other joint geometries without further modification. Crocombe and co-workers have extended this approach to predict the residual strength and service lifetime of joints degraded by moisture and fatigue loading [6-9]. In contrast to the above and other studies, all of which apply the model to bonded joints with conventional epoxy based adhesives, little has been reported on joints with a low modulus and thick adhesive layer. Loureiro [10] et al have modelled a T-Peel joint with a flexible but thin polyurethane adhesive layer using cohesive zone elements. It seems likely that progressive damage modelling of the joint bonded with a flexible and thick adhesive layer might be potentially challenging because the large deformations could lead to a greater risk of adverse mesh distortion and hence solution convergence difficulties.

Thus, this part 2 of the paper investigates the application of such modelling procedures to thicker and more flexible adhesive systems. Cohesive zone parameters were fitted using the experimentally measured load-displacement responses corresponding to the pure mode I and the mode II loading discussed in the partner paper. Using the fitted cohesive zone material models, progressive damage modelling of two joints representative of those used in the vehicle structure, was carried out. A peel joint and a lap shear joint were chosen by the manufacturer as representative joints as i) they comprise the same, dissimilar substrate materials as used in the vehicle joints, ii) are subjected to mixed mode loadings typically experienced by the vehicle in operation and iii) use the same adhesive and (large) adhesive thickness that are used in the vehicle. The numerical predictions were compared with the experimental results and the validity of the predictions was assessed from both a quantitative and a qualitative point of view. It should be noted that in this work the authors have deliberately used the same thickness of adhesive in the tests to determine the key fracture parameters as the thickness in the structural joints being assessed. This is generally good practice but, due to the high ductility of the adhesive resulting in an extensive damage zone, the same fracture parameters may not be as applicable to structural joints with other thickness adhesive layers. This is an area that should be explored in future work.

\section{Determination of cohesive zone parameters}

\subsection{The FE models}

\subsubsection{Mode I}

Two dimensional FE modelling with cohesive elements using the FE code ABAQUS was performed to calibrate the mode I cohesive parameters. Since it was known the adhesive shows a fairly linear relationship in a shear (in the thick adherend shear test, TAST), with no extended stress plateau, a triangular rather than trapezoidal traction-separation law was selected for use in the FE modelling, see Figure 1. The only unknown parameter was the maximum traction in mode I, $\sigma_{\max }$, since the fracture energy was obtained from the testing detailed in part 1 of the paper [1]. The DCB test data for the specimens with $6 \mathrm{~mm}$ thick substrates showed relatively consistent crack growth in the adhesive layer and hence was selected for the calibration modelling because it exhibited the same failure mode as shown in 
the representative peel joint test, described in Section 3 of this paper. The model geometry is shown in Figure 2 along with the boundary conditions. The loading blocks on the FE model were subjected to a displacement cycle up to $18 \mathrm{~mm}$, down to $0 \mathrm{~mm}$, and then up again to 60 $\mathrm{mm}$. This corresponded to the pre-cracking, unloading and the re-loading in the experimental tests. Horizontal movement of the loading points was constrained. Instead of modelling the adhesive as a single layer of cohesive elements as is often done for thin bondlines, elastic adhesive layers are modelled separately adjacent to the cohesive elements. The cohesive elements are $0.05 \mathrm{~mm} \times 0.1 \mathrm{~mm}$ and are located at the interface with the upper substrate, running from the tip of the pre-crack to the far end of the specimen. The cohesive layer was connected to the adhesive and the substrate elements using tie constraints. Four-node linear plane strain hybrid incompatible elements (CPE4HI) were used for the adhesive, whilst 4node linear incompatible plane stress elements (CPS4I) were used for the substrate. It should be noted that, in spite of the large bond thickness, a preliminary 3D stress analysis indicated that the bond layer was strongly constrained by the substrates and was essentially uniformly in plane strain state through the thickness.

The composite substrates (with a random orientation of chopped fibres) were modelled with isotropic linear material properties, having a Young's modulus of $11.4 \mathrm{GPa}$ and a Poisson's ratio of 0.3 . The linear material properties used for the adhesive were a shear modulus of 2.8 $\mathrm{MPa}$ and a Poisson's ratio of 0.45 . The value of shear modulus was obtained from thick adherend shear tests (TAST). The substrates were steel with a $10 \mathrm{~mm}$ overlap length, $4 \mathrm{~mm}$ overlap substrate thickness and the same adhesive thickness as the other joints tested $(3 \mathrm{~mm})$. Finite element modelling of this configuration showed that the shear stress was constant over most of the overlap length. These joints were tested at a cross-head speed of $1 \mathrm{~mm} / \mathrm{min}$ (consistent with the other configurations tested). A typical shear stress-strain plot is shown in Figure 3 and can be seen to be largely linear over most of the loading history.

The same adhesive elastic properties were assigned to the cohesive elements, and the mode I fracture energy of $5.7 \mathrm{~kJ} / \mathrm{m}^{2}$, experimentally measured for the $6 \mathrm{~mm}$ substrate DCB specimens, was used. Several $\sigma_{\max }$ values were assessed to obtain the best fit to the experimental DCB load-displacement curve.

\subsubsection{Mode II}

The experimental data from the cracked TAST fracture tests were used to evaluate the maximum shear traction in mode II, $\tau_{\max }$, by conducting two dimensional FE modelling with ABAQUS. The same test data used in the computation of the mode II fracture energy (Part 1) was used for this calibration work. The FE model is shown in Figure $\mathbf{4}$ together with the boundary conditions. One end of the steel substrate was fixed in all directions, and a displacement corresponding to that at the failure of the specimen was applied at the other end. Cohesive layers with the same element size as used in the DCB calibration were located on both adhesive substrate interfaces with a $7 \mathrm{~mm}$ pre-crack being modelled for one side. Tie constraints were used between the cohesive layer and the adjacent substrate and adhesive elements. The crack path was based on the assumption that the pre-crack had been diverted to the interface at the peak load measured in the test, as discussed in the Part 1 paper. The elements used for the adhesive, the substrate, and the cohesive layer were the same as in the DCB model, as were the adhesive properties. Linear material properties including a Young's modulus of $200 \mathrm{GPa}$ and Poisson's ratio of 0.3 were used for the steel substrates. The cohesive elements were assigned the same elastic properties as the adhesive, and the mode II fracture energy of $11.6 \mathrm{~kJ} / \mathrm{m}^{2}$ determined in Part 1, was used. Several $\tau_{\text {max }}$ values were assessed to determine the best fit to the experimental load-displacement curve. 


\subsection{Results and discussions}

\subsubsection{Mode I}

The load-displacement curves obtained as a result of the modelling for three different values of $\sigma_{\max }$ are shown in Figure 5 together with the experimental curve for pre-cracking. It can be seen that the peak load and the non-linearity near the peak load are significantly affected by $\sigma_{\max }$. A value of $4 \mathrm{MPa}$ for $\sigma_{\max }$ accurately represents the loading response and thus was used in the subsequent modelling of representative joints. Figure 6 compares modelling results for both the pre-cracking and the re-loading phase with the experimental results. The modelling results successfully predicted the marked increase in the compliance during the re-loading, due to the slight damage growth and the considerable process zone caused by pre-cracking, in addition to the gradual load decrease during the crack propagation. Furthermore, Figure 7 shows the good agreement between the predicted and measured crack growth, suggesting that the model can represent the entire progressive damage behaviour well. From these results, it is suggested that pre-cracking load curve should be used to fit $\sigma_{\max }$ for adhesives that exhibit a considerable process zone because the peak load and the compliance in the re-loading phase might already be affected by the damage process which occurred during the pre-cracking phase.

\subsubsection{Mode II}

The predicted load-displacement curves for three different values of $\tau_{\max }$ are shown in Figure $\mathbf{8}$ and compared with the experimental curve. It can be seen that $\tau_{\max }$ has a remarkable impact on both the peak load and the displacement at failure, with a higher $\tau_{\max }$ enhancing both. Regardless of the $\tau_{\max }$ values, the predicted stiffness was slightly lower than the experimental results. This possibly arises from the non-linear elasticity typical of rubbers, becoming stiffer especially at lower and higher strains. The difference from the actual stiffness in the small displacement region appears to have caused the gap between the predicted and experimental loads. Neglecting this small stiffness difference, a value for $\tau_{\max }$ of $6.5 \mathrm{MPa}$, which shows a similar peak load and slightly larger displacement at failure, was regarded to give the best fit and thus used in the subsequent modelling of the representative joints. Having a higher $\tau_{\max }$ than $\sigma_{\max }$ may at first sight appear odd, however, the physical meaning of the maximum tractions is rather ambiguous [11]. Besides, if molecular bond breakage under tensile force is the primary damage initiation mechanism, as is generally supposed for rubbers [12], then the critical tensile stress causing damage is the same as the shear stress in a pure shear state, and thus the critical stresses in pure mode I and mode II are likely to be at least of the same order (and mode I may be lower due to the effect of hydrostatic stress).

\section{Prediction of the structural response of representative joints}

\subsection{Peel joint}

\subsubsection{Experimental methods}

The peel test specimen geometry is shown in Figure 9. A $90^{\circ}$ peel joint was considered to be most suitable considering the rigidities of the dissimilar substrates, i.e. the GFRP composite does not show enough ductility to form the flexible part of the joint, and the steel would be difficult to use in a $180^{\circ}$ peel joint. The steel substrate took the same thickness as used in the actual joints on the vehicle. Other dimensions of the joint were specified estimating critical adhesive stresses and load using a closed form analysis based on the Bigwood and Crocombe approach $[13,14]$ so that premature failure of the composite substrate can be avoided. The two substrates were bonded with the target polyurethane adhesive having a $3 \mathrm{~mm}$ thick bondline. No adhesive fillet was created at either end of the overlap. A paper scale and 
typewriter correction fluid were applied to visually assess the crack propagation as was done in the fracture testing reported in the Part 1 paper.

The specimen was fixed in an Instron universal testing frame by directly clamping the composite substrate onto the cross head $10 \mathrm{~mm}$ away from the both ends of the bond overlap. The vertical part of the steel substrate was clamped in a rigid mechanical grip $100 \mathrm{~mm}$ above the bondline. The specimen was loaded at a constant cross-head rate of $1 \mathrm{~mm} / \mathrm{min}$ until separation of the substrates occurred. It should be noted that as the same cross-head rate was used in all four test configurations reported in this paper, due to the geometry of the specimens this has resulted in times to reach peak load which range from 4 mins in the TPeel to 15 mins in the mode I fracture tests. However, in these tests the actual crack growth rates were similar, $1.25 \mathrm{~mm} / \mathrm{min}$ and $1.7 \mathrm{~mm} / \mathrm{min}$. This suggests that any strain rate effects should not be significant. During the test the crack propagation was periodically captured using a high resolution camera connected to the display of a PC and correlated with the load and displacement. Testing was carried out on five specimens.

\subsubsection{Modelling methods}

The quasi-static mechanical response of the peel joint was modelled with the FE code ABAQUS using cohesive elements to provide progressive damage in the adhesive layer. As will be described later, the crack growth observed in the testing was primarily twodimensional in nature. This suggested that it would be appropriate to use a two-dimensional FE analysis. The model of the peel joint along with boundary conditions is shown in Figure 10. A vertical displacement was applied at the upper end of the steel substrate, with its horizontal movement being fixed. All displacements were constrained at the positions the substrate were clamped in the testing. Based on experimental observations detailed later, cohesive elements of the same size as used in the calibration modelling discussed above $(0.05$ $\mathrm{mm} \times 0.1 \mathrm{~mm}$ ) were located on the upper interface, with tie constraints to the adjacent substrate and adhesive.

The same element types were used as in the determination of the cohesive parameters, namely CPE4HI elements for the adhesive and CPS4I elements for the substrates. Also, the same linear material properties were used for the materials. However, unlike the modelling work for fitting the mode II cohesive parameter, elastic-plastic properties were assigned to the steel substrate (with the von Mises yield criterion and isotropic strain-hardening), to take into account the significant plastic deformation observed in the testing. The hardening slope of the steel, obtained elsewhere, is shown in Figure 11. It should be noted that the calibrated cohesive parameters for mode I and mode II, determined in the previous sections, were applied without further modification. A quadratic relationship between the tractions given below was applied as a mixed mode damage initiation criteria.

$$
\left\{\frac{\sigma}{\sigma_{\max }}\right\}^{2}+\left\{\frac{\tau}{\tau_{\max }}\right\}^{2}=1
$$

where $\sigma$ and $\tau$ are the normal and shear tractions acting on the cohesive elements, respectively. For crack propagation, the simple mixed mode fracture criterion developed in the Part 1 paper was incorporated, i.e. 


$$
\left\{\frac{G_{I}}{G_{I C}}\right\}^{1.5}+\left\{\frac{G_{I I}}{G_{I I C}}\right\}^{1.5}=1
$$

A schematic diagram of the mixed-mode cohesive zone model is shown in Figure 12, where $T, \delta_{n}$ and $\delta_{s}$ denote traction and displacement in normal and shear direction, respectively.

\subsubsection{Results and discussions}

A representative experimental load-displacement curve along with the observed crack length is shown in Figure 13. The initial loading increase showed a slight non-linearity as the crack initiated in the adhesive layer beneath the loading point. However, the initiated crack locations in the adhesive thickness varied between the specimens, some showing the initiation close to the upper interface and others close to the lower interface. One specimen showed cracking just within the composite substrate surface which resulted in a significantly lower maximum load. However, in all cases, the initiated crack propagated towards the upper interface as shown in Figure 14 (the specimen with the initial failure locus on the lower interface) as this is likely to be the direction of maximum mode I energy release rate. This then grew stably near the interface, corresponding to the relatively uniform plateau region in the load curve. This consistent crack growth is considered to be caused by the high deformability of the steel substrate compressing the highly stressed adhesive zone close to the crack tip as observed in the DCB testing with thinner substrates (see section 2 of the Part 1 paper). Except for the specimen that showed initial failure within the composite substrate the failure mode was predominantly cohesive in the adhesive.

Figure 15 shows the upper and lower bounds of the experimental load-displacement responses together with the modelling results. The numerical prediction successfully reproduces the initial load peak associated with crack initiation phase. However, the gradual load decrease afterwards, seen in the modelling results, is in contrast to the experimental trend where load was retained or even increased with longer crack propagation. Moreover, the plastic deformation of the steel substrate as well as the crack propagation behaviour was not completely captured. Figure 16 represents the numerical and experimental deformed shape at the same displacement of $56 \mathrm{~mm}$. Although there are clear similarities between the FE modelling and the experimental data and the FE model has simulated extensive damage and deformation in both the steel and the adhesive, there are some discrepancies in the deformed steel shape and the crack length between the two. The peeled portion of the steel appears to be unusually elongated compared to the crack length. The fully damaged cohesive elements still seem to constrain the peeled steel, preventing its lateral movement. It is highly probable that the predicted gradually decreasing load with the crack propagation mentioned earlier is caused by this behaviour. This numerical problem unfortunately made it difficult to carry out further quantitative or qualitative evaluation of the progressive damage modelling with regard to the structural response of the peel joint and is an area that will be studied further in future work.

\subsection{Lap shear joint}

\subsubsection{Experimental methods}

The dimensions of the lap shear joint, using the same dissimilar substrate materials as used in the vehicle joints, are shown in Figure 17. The steel substrate thickness $(1.5 \mathrm{~mm})$ was the same as used in the vehicle. A closed form analysis, based on the same method used for the peel joint, was performed to check that the composite substrate would have enough margin against premature failure. The two substrates were bonded with the target adhesive having a 3 $\mathrm{mm}$ thick bondline. Aluminium tabs were bonded on both sides of the substrate ends to enable 
load alignment and avoid the need to grip the GFRP directly. As in the peel joint, correction fluid and a paper scale were applied to facilitate observation of adhesive cracking.

The specimen was placed in an Instron servo-mechanical testing frame using mechanical wedge action grips. Other procedures were as outlined in the section above on peel testing. Five replicates were tested.

\subsubsection{Modelling methods}

Figure 18 illustrates the model for the lap shear joint. Both upper and lower surfaces of the tabbed ends were constrained against movement in the vertical direction. The end of the composite substrate was given a horizontal displacement whilst at the other end the tabs were fixed in the horizontal direction. The cohesive elements were the same size as those used in the peel joint and mode I and II fracture test models and were located on both the upper and lower interfaces based on the locus of failure observed in the testing. Tie constraints were used to attach the cohesive elements to the adjacent substrate and adhesive. Also, the same element types and material properties (including the cohesive zone parameters) used in the peel joint modelling were used in this single lap joint modelling.

\subsubsection{Results and discussions}

A typical experimental load-displacement curve for the lap shear joint is shown in Figure 19. Crack initiations were observed at the overlap ends before the maximum load was reached, but in various locations over the width of the joint. The crack propagated slowly along one or both interfaces, as shown in Figure 20, up to the point when unstable crack propagation with a steep drop in the load occurred. The considerable rotation of the overlap region arising from the offset forces caused by the highly asymmetric joint configuration can also be seen in the same figure. The specimens showed predominantly cohesive failure.

Figure 21 shows a comparison between the load-displacement response predicted by the progressive damage modelling and the upper and lower bounds of the experimental data. The trend of the curve is captured by the modelling reasonably well. Figure 22 presents damage contours for damage initiation (Figure 22 (a)) as well as when the damage was fully developed (Figure 22 (b)), and the loads at which both images are taken are indicated on Figure 21. It can be seen that damage was predicted to initiate at the tensile strained overlap ends at an early stage, however, it was not until immediately before the peak load that the damage was fully developed at the end. This early damage initiation and slow propagation arose from the relatively low maximum tractions and substantially high fracture energies, and is qualitatively in accordance with the experimental observations described above. As with the mode II fracture testing, the numerical results showed a slightly lower stiffness. Again, this might be attributed to the nonlinear elasticity of the adhesive. Indeed, the stiffness for the experimental load curves appears to increase at larger displacements, close to the peak load. However, since the difference in the stiffness can be seen over the entire initial loading stage, this might not be the only cause. Figure $\mathbf{2 3}$ presents the numerically predicted deformation and stress states of the substrates immediately before the steep load decrease. It is found that the steel substrate exceeds the stress level required to cause plastic deformation. In fact, permanent deformation was recognised in the specimen after testing. By comparing with Figure 20, it is demonstrated that the structural response of the joint is qualitatively well captured by the numerical modelling. However, it can be seen in the figure that the composite substrate is more deformed in the modelling results. This might arise from slight differences in the boundary conditions between the actual testing and modelling. Although the movements of the aluminium tabs perpendicular to the loading direction were completely constrained in the model, the mechanical wedge grip was able to rotate a little. This could relieve the deformation of the substrate to some extent. 


\section{Conclusions}

The structural response of the peel and lap shear joints bonded by an automotive structural adhesive system with similar geometric features to the vehicle joints were predicted by progressive damage modelling. Appropriate mode I and II parameters for the progressive damage cohesive zone elements were a) obtained directly from the fracture mechanics testing reported in Part 1 (fracture energies) and b) were calibrated by matching the loaddisplacement curves of the FE predicted and experimental mode I and mode II fracture test results (critical tractions). It was found that the traction value for mode I significantly affected the maximum load, whilst for mode II it has prominent influence on both the maximum load and displacement to failure. From this calibration the maximum traction for mode II (6.5 $\mathrm{MPa})$ was slightly higher than that for mode I (4 MPa). Using these calibrated cohesive parameters, without further modification, and the mixed mode fracture criterion developed in the Part 1 paper, the testing results for the peel and lap shear joints were predicted. Reasonable correlation was achieved between the numerical and experimental results for the peel joint in spite of the extensive plastic deformation the steel substrate experienced. For the lap shear joint, comparisons of the numerical prediction with the testing resulted in good agreement with the experimental load-displacement relationships. Further, the modelling results matched the damage growth behaviour observed in the tests. Based on these results, it can be stated that the modelling approach presented can be applicable to bonded joints with a rubbery and thick adhesive layer.

\section{References}

[1] Hasegawa K, Crocombe AD, Coppuck F, Jewel D and Maher S, International Journal of Adhesion and Adhesives, under review.

[2] de Moura, MFSF, 'Progressive Damage Modelling'. In:da Silva LFM and Ochesner A (eds.), Modelling of adhesively bonded joints, Verlag: Springer, p. 155-182, 2008.

[3] Yang, Q. D. and Thouless, M. D. 'Numerical simulations of adhesively-bonded beams failing with extensive plastic deformation' Journal of Mechanics and Physics of Solids, 47: $1337-1353 ; 1999$.

[4] Yang, Q. D. and Thouless, M. D. 'Elastic-plastic mode-II fracture of adhesive joints', International Journal of Solids and Structures, 38: 3251-3262; 2001.

[5] Yang, Q. D. and Thouless, M. D. 'Mixed-mode fracture analysis of plastically deforming adhesive joints', International Journal of Fracture, 110: 175-187; 2001.

[6] Liljedahl C. D. M, Crocombe A. D., Wahab, M. A. and Ashcroft, I. A. 'Modelling the environmental degradation of adhesively bonded aluminium and composite joints using a CZM approach’ International Journal of Adhesion and Adhesives, 27: 505-518; 2007.

[7] Hua, Y., Crocombe, A. D., Wahab, M. A. and Ashcroft, I. A. 'Continuum damage modelling of environmental degradation in joints bonded with EA9321 epoxy adhesive' International Journal of Adhesion and Adhesives, 28: 302-313; 2008.

[8] Khoramishad, H., Crocombe, A. D., Katnam, K. B. and Ashcroft, I. A. 'Fatigue damage modelling of adhesively bonded joints under variable amplitude loading using a cohesive zone model' Engineering Fracture Mechanics, 78: 3212-3225; 2011. 
[9] Sugiman, S., Crocombe, A. D. and Ashcroft, I. A. 'The fatigue response of environmentally degraded adhesively bonded aluminium structures' International Journal of Adhesion and Adhesives, 41: 80-91; 2013.

[10] Loureiro, A. L., da Silva, L. F. M., Sato, C. and Figueiredo. M. A. V. 'Comparison of the mechanical behaviour between stiff and flexible adhesive joints for the automotive industry' Journal of Adhesion, 86: 765-787; 2010

[11] Blackman, B. R. K., Hadavinia, H., Kinloch, A. J. and Williams, J. G. 'The use of a cohesive zone model to study the fracture of fibre composites and adhesively-bonded joints', International Journal of Fracture, 119: 25-46; 2003.

[12] Kinloch, A. J. and Young, R. J. Fracture behaviour of polymers. London: Elsevier Applied Science; 1983.

[13] Bigwood DA and Crocombe AD 'Elastic analysis and engineering design formulae for bonded joints', Intl J Adhesion and Adhesives, 9: 229-242; 1989

[14] Crocombe, A. D. and Ashcroft, I. A. 'Simple lap joint geometry' in: da Silva, L. F. M. and Ochesner, A. (eds.) Modelling of adhesively bonded joints. Verlag: Springer. p. 3-23; 2008. 


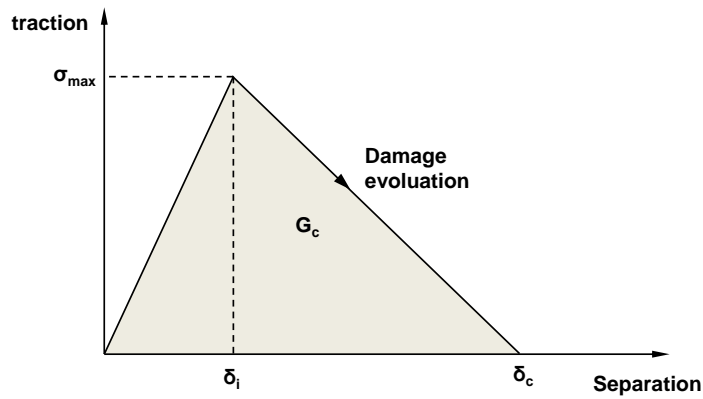

Figure 1 A triangular traction-separation law

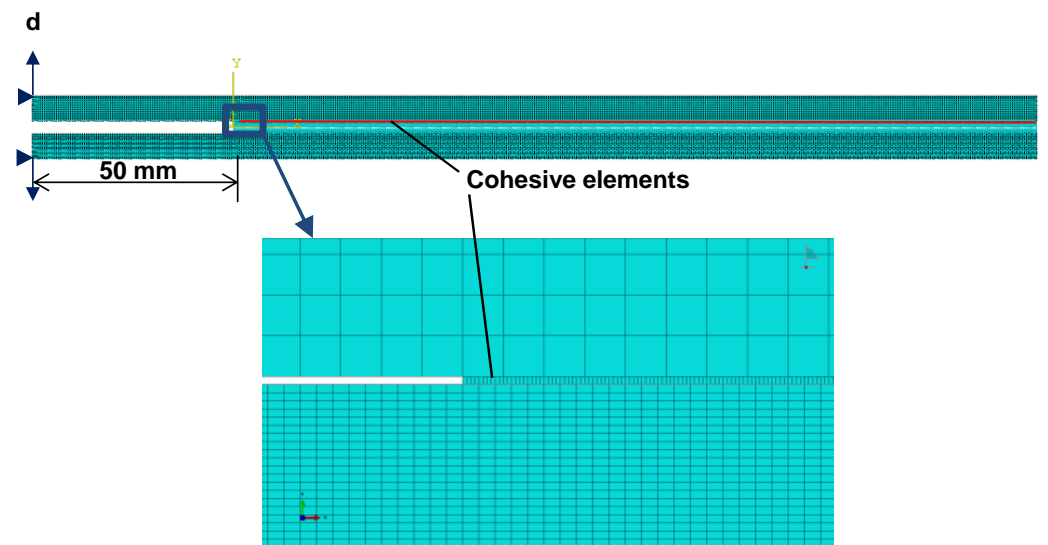

(Unit: $\mathrm{mm}$ )

Figure 2 FE model geometry of DCB specimen.

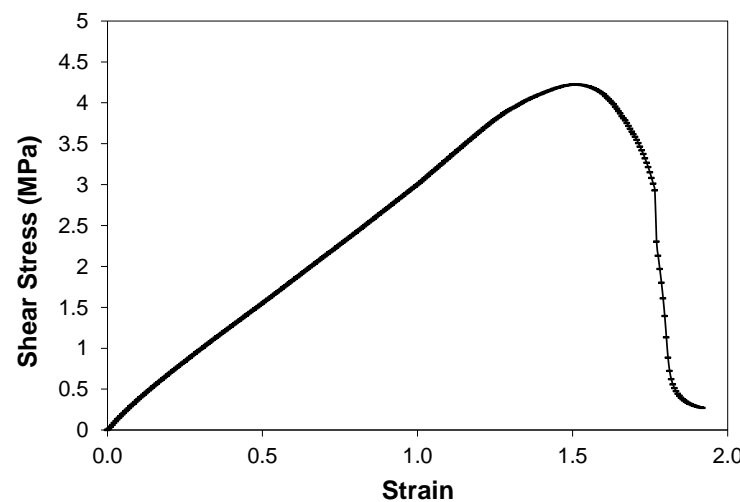

Figure 3 A typical shear stress-strain response of the adhesive obtained by a TAST.

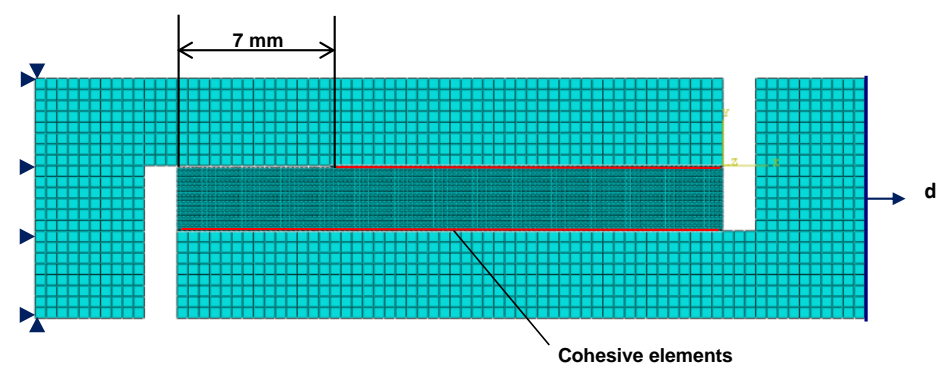

Figure 4 FE model geometry of the cracked TAST specimen. 


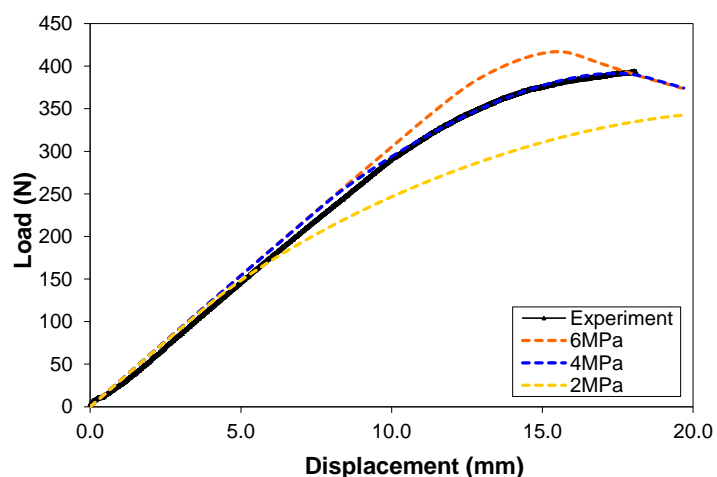

Figure 5 Comparison of predicted Mode I load-displacement curve for three different $\sigma_{\max }$ of $2 \mathrm{MPa}, 4 \mathrm{MPa}$, and $6 \mathrm{MPa}$ with experimental results during the pre-cracking.

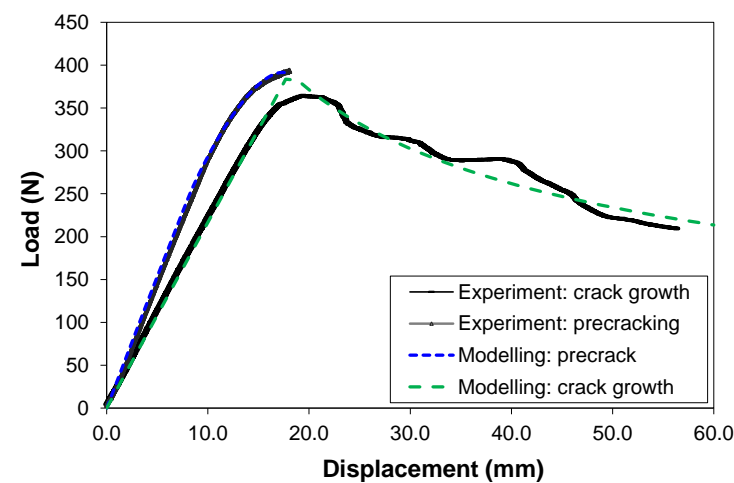

Figure 6 Comparison of predicted load-displacement curve with experimental results during the re-loading of the Mode I specimen.

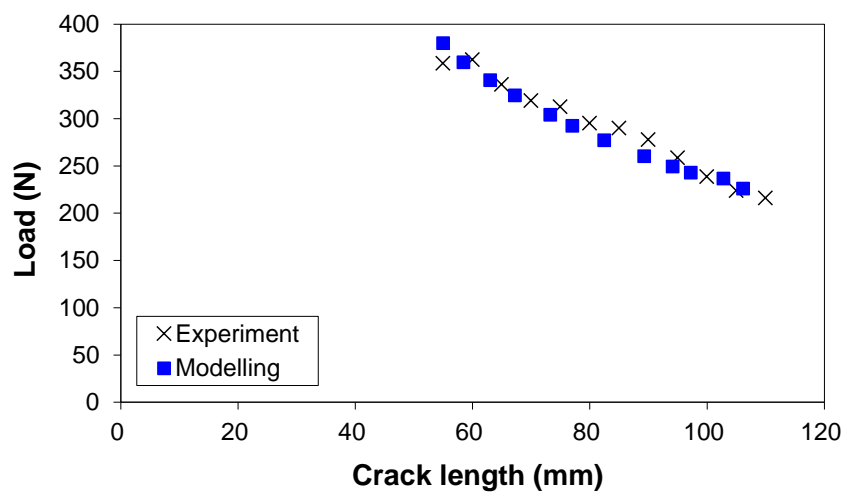

Figure 7 Comparison of predicted and experimentally measured crack length of the Mode I specimen. 


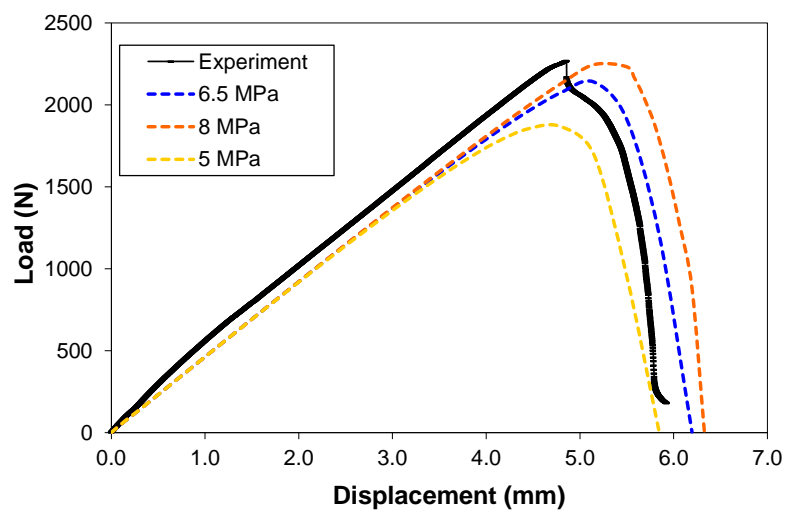

Figure 8 Comparison of predicted load-displacement curve for three different $\tau_{\max }$ of $5 \mathrm{MPa}, 6.5 \mathrm{MPa}$, and 8MPa with the experimental results for the Mode II specimen.

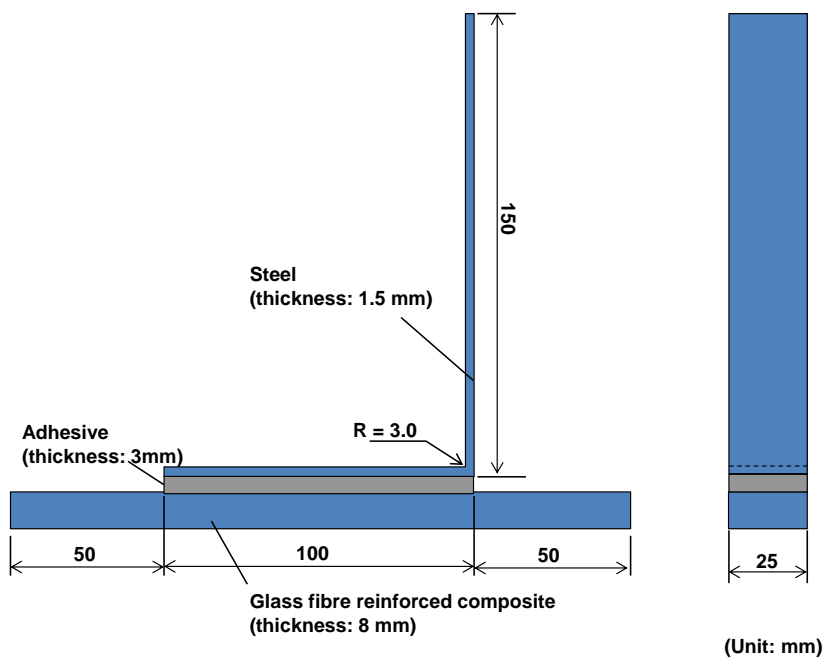

Figure 9 Peel test specimen geometry. 


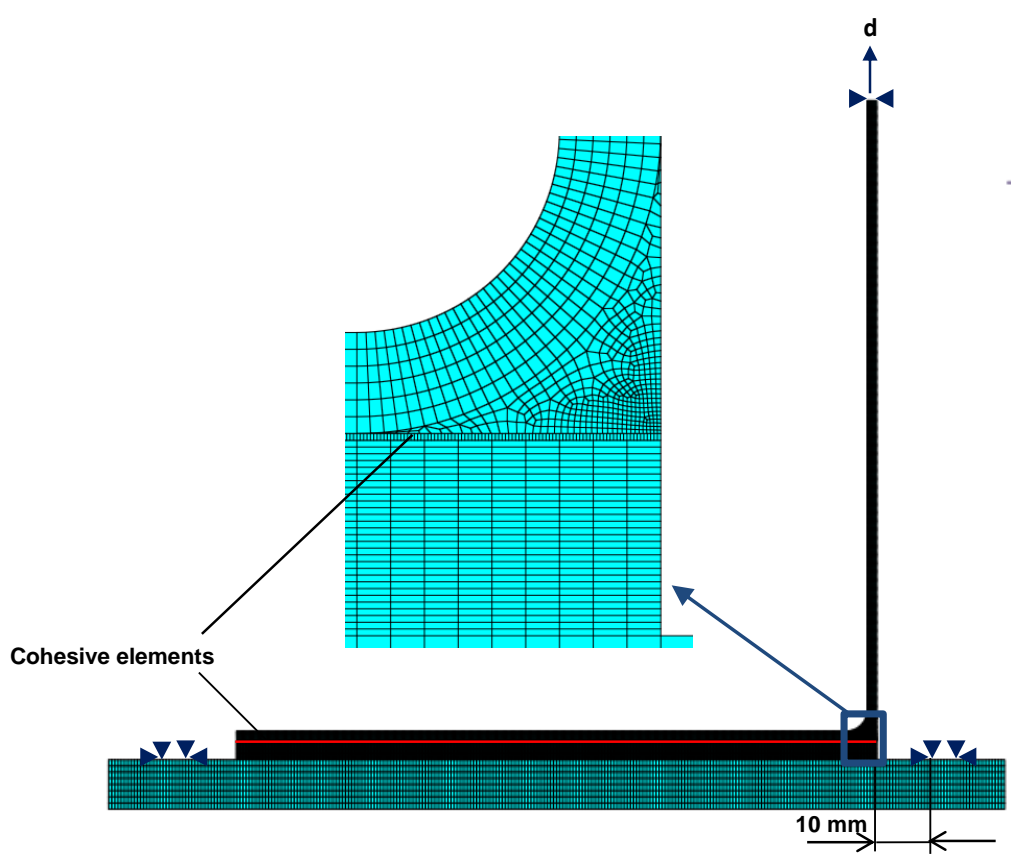

Figure 10 FE model geometry of the peel specimen with cohesive elements along the interface.

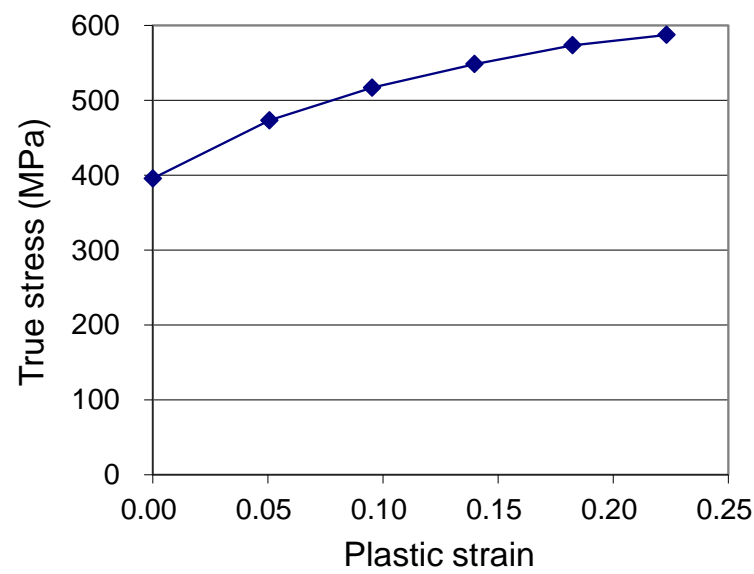

Figure 11 Strain-hardening slope for the substrate steel. 


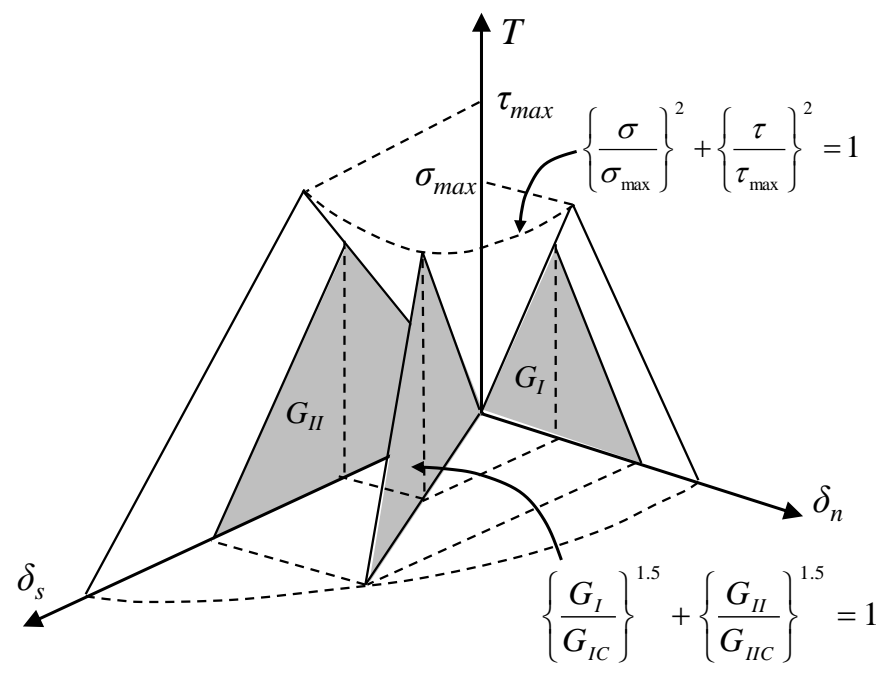

Figure 12 Schematic representation of the mixed-mode cohesive zone model.

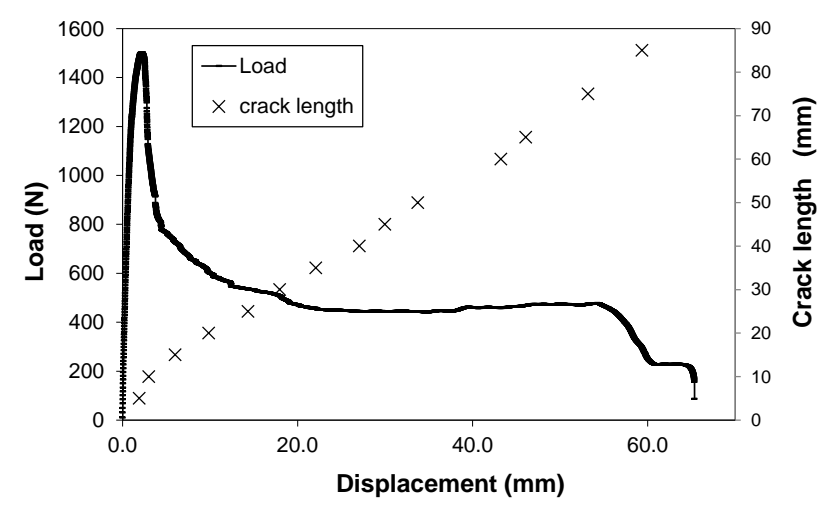

Figure 13 Typical load-displacement curve for the peel specimen along with crack length.

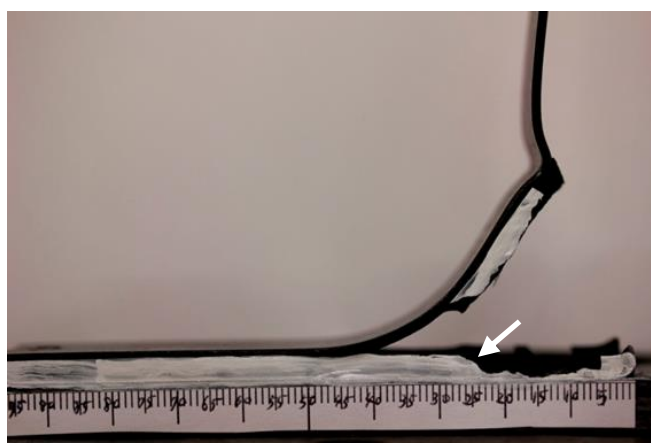

Figure 14 Crack growth behaviour of the peel specimen at displacement of $27 \mathrm{~mm}$ the load curve indicated in Figure 10. 


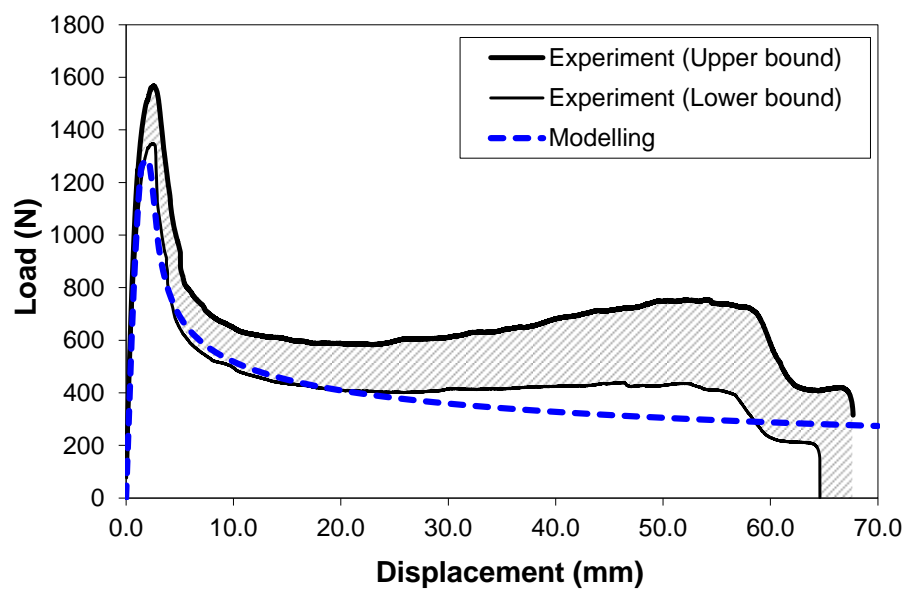

Figure 15 Comparison of predicted and experimentally measured structural responses of the peel joints.
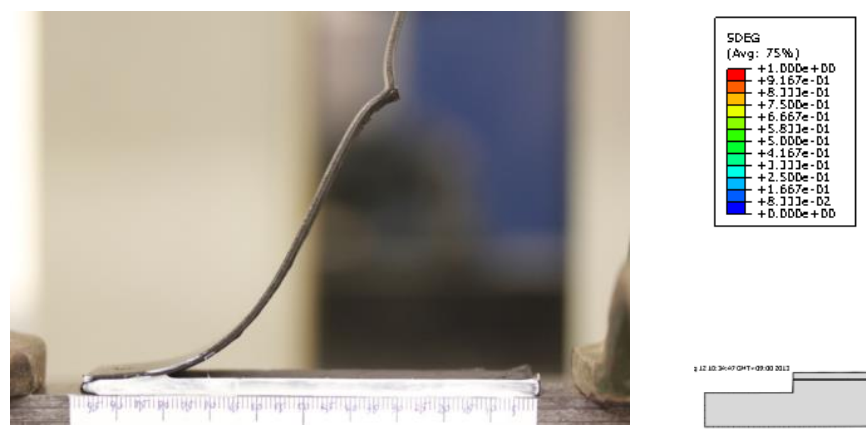

Figure 16 Comparison of the steel deformation and crack propagation state at a displacement of $56 \mathrm{~mm}$ between experimental and modelling results.

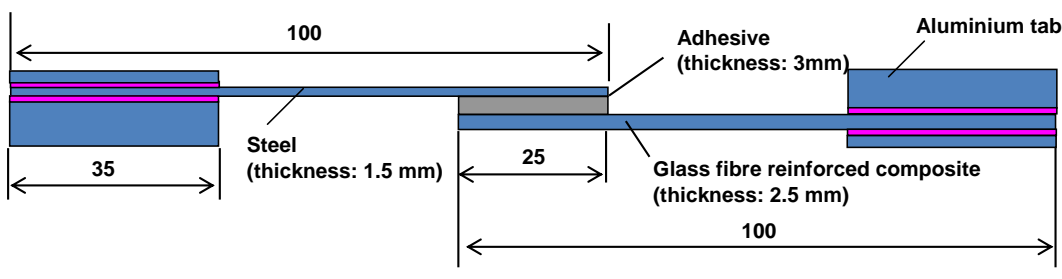

(Unit: mm)

Figure 17 The Lap shear test specimen geometry. 


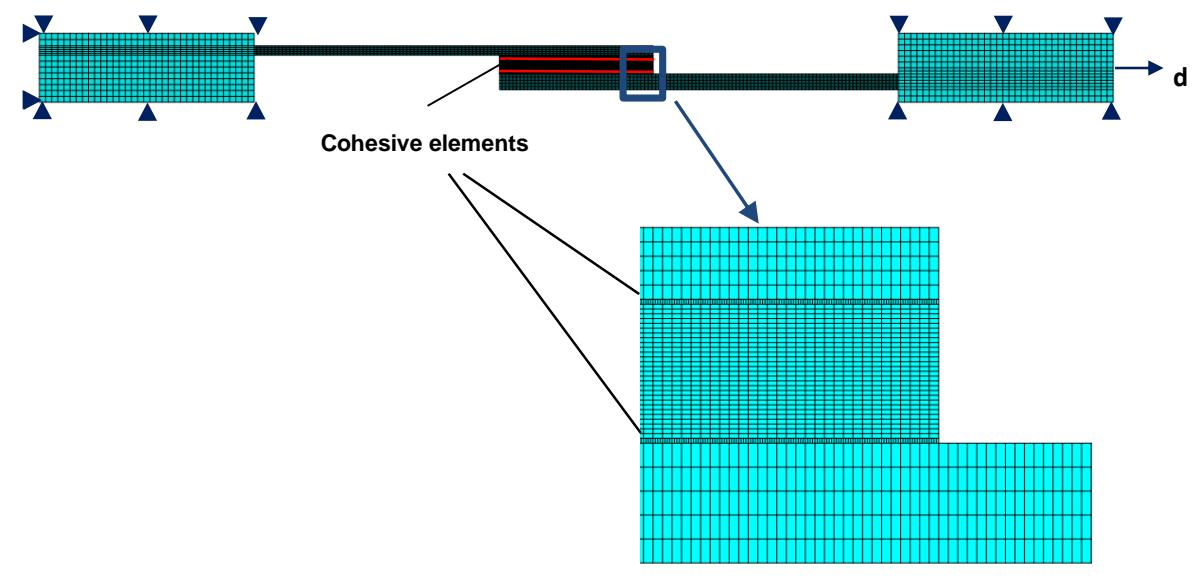

Figure 18 FE model geometry of lap shear specimen with cohesive elements along the both upper and lower interfaces.

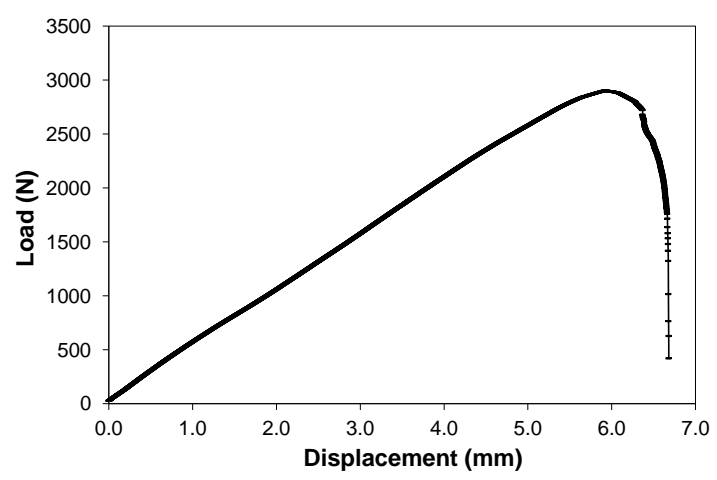

Figure 19 Typical load-displacement curve for the lap shear specimen.

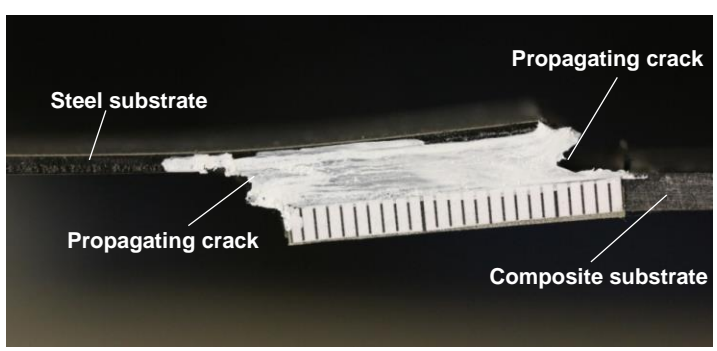

Figure 20 Crack propagation of the lap shear specimen before the steep decrease in the load indicated in Figure 17. 


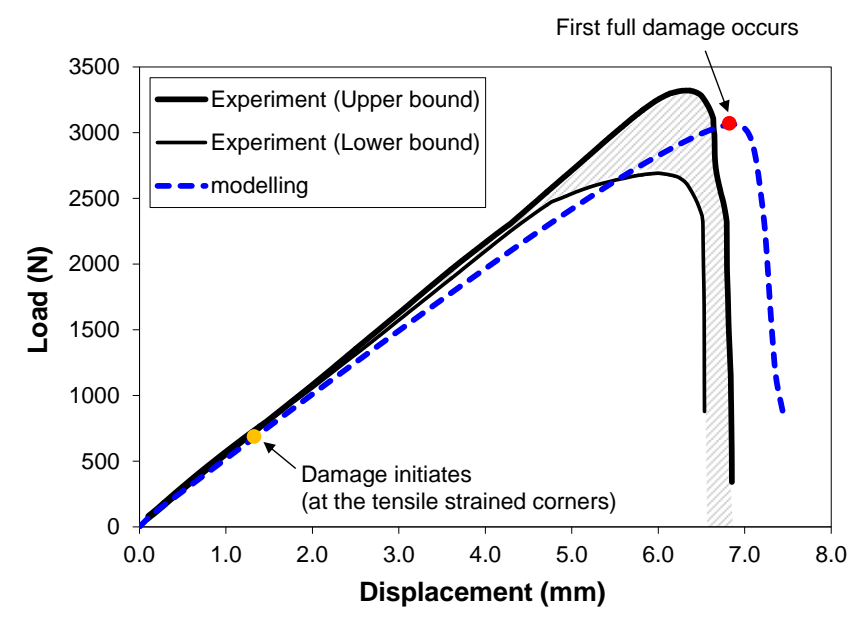

Figure 21 Comparison of predicted and experimentally measured structural response of the lap shear joints.

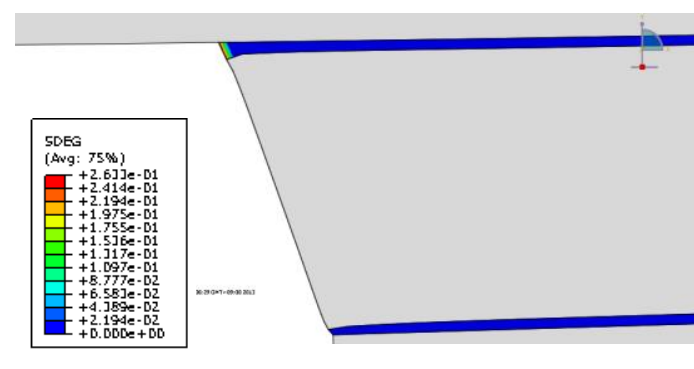

(a)

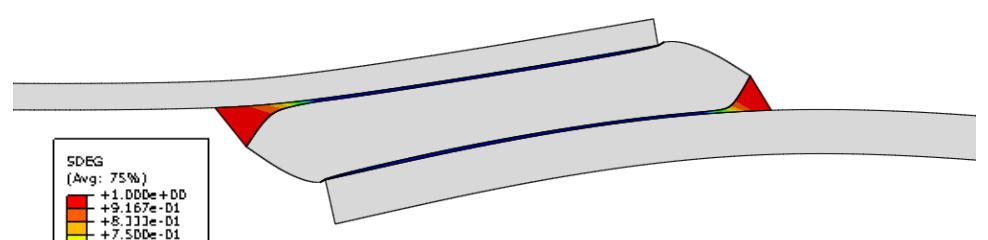

(b)

Figure 22 Deformation and damage state for the lap shear specimen: (a) when damage initiates (b) when first full damage occurs (see Fig 19 for location son the load-displacement curve).

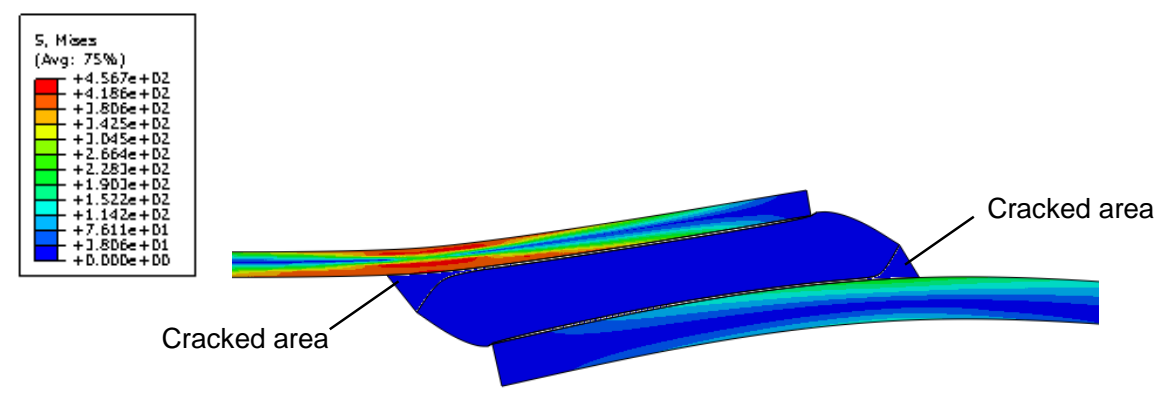

Figure 23 Deformation, stress and damage state for the lap shear specimen immediately before the steep load decrease. 